\title{
CRITÉRIOS PARA ALOCAÇÃO DE RECURSOS ESCASSOS - LEITOS HOSPITALARES - EM VISTA DO COVID-19
}

Gabriel Schulman

Advogado. Sócio de Trajano Neto e Paciornik. Doutor em Direito (UERJ). Mestre em Direito (UFPR). Especialista em Direito (Coimbra). Professor da Universidade Positivo. E-mail: gabriel.schulman@tnp.adv.br.

\section{Consulta}

Diante da crise sanitária no contexto do Covid-19, o Grupo Médico XXX apresenta consulta acerca de critérios de alocação e/ou priorização de leitos de internação hospitalar, sobretudo em unidade de tratamento intensivo (UTI).

\section{Contextualização}

No mundo todo, a escalada do Covid-19 impôs a necessidade de enfrentar escolhas difíceis (e trágicas) em matéria de alocação de recursos de atenção à saúde. ${ }^{1}$ Em nosso país, a realidade infelizmente é a mesma, ou quiçá ainda mais grave, afinal "O Brasil já enfrentava escassez de leitos de UTI no SUS antes do novo coronavírus chegar". ${ }^{2}$ No cenário nacional, observa-se acentuada escalada

ROSENBAUM, L. Facing Covid-19 in Italy - Ethics, logistics, and therapeutics on the epidemic's front line. New England Journal of Medicine, v. 382, n. 21, p. 1873-1875, 2020. Todos os artigos deste parecer foram acessados novamente na véspera de sua conclusão, em 4.4.2021.

2 COLLUCCI, Cláudia. Algoritmos e inteligência artificial podem ajudar Brasil a decidir sobre leitos de UTI. Folha de S.Paulo, São Paulo, 24 mar. 2020. Disponível em: https://www1.folha.uol.com.br/equili brioesaude/2020/03/algoritmos-e-inteligencia-artificial-podem-ajudar-brasil-a-decidir-sobre-leitos-de-uti. shtml?origin=uol. Acesso em: 4 abr. 2021. 
do Covid-19, com escassez de recursos de atenção à saúde de todos os tipos, inclusive equipamentos, ${ }^{3}$ oxigênio, ventiladores e leitos, como denotam as recentes manchetes: "SP: ocupação de UTI-Covid do SUS em cidades do interior passa dos 100\%",4 "16 estados têm ocupação de mais de 90\%, três não possuem mais vagas", 5 "SUS nos estados não tem leitos de UTI contra o coronavírus", 6 "País tem mais de mil cidades com dificuldades no abastecimento de oxigênio", "Diante de colapso, Ribeirão Preto não tem condições de abrir mais UTIs para Covid, diz Prefeitura: 'Estamos no limite'”, , “Ameaça à saúde pública global': colapso dos hospitais no Brasil é destaque na mídia estrangeira”. ${ }^{9}$

Diante deste cenário, apresenta-se o dilema ético da alocação de recursos escassos. Os parâmetros para internação de pacientes, sobretudo em unidade de terapia intensiva não estão definidos de modo claro e a falta de recursos decorre de fatores sobretudo externos aos médicos, no entanto, estabelecer os parâmetros mostra-se fundamental para atender aos desafios que a crise sanitária em curso nos impõe. Verifica-se desta maneira um cenário de "desequilibrio entre las necesidades clínicas y la disponibilidad efectiva de los recursos sanitários”.10

3 A própria otimização de equipamentos é preconizada pela Organização Mundial da saúde (WHO. Rational use of personal protective equipment for coronavírus disease 2019 (Covid-19): interim guidance. 27 fev. 2020. Disponível em: https://apps.who.int/iris/bitstream/handle/10665/331215/WHO2019-nCov-IPCPPE_use2020.1-eng.pdf. Acesso em: 4 abr. 2021).

4 CÉSAR, Daniel. SP: ocupação de UTI-covid do SUS em cidades do interior passa dos 100\%. UOL, Pereira Barreto, 3 abr. 2021. Disponível em: https://noticias.uol.com.br/saude/ultimas-noticias/ redacao/2021/04/03/sp-ocupacao-de-uti-covid-do-sus-em-cidades-do-interior-passa-dos-100.htm. Acesso em: 4 abr. 2021.

5 SOUZA, Paloma; GALZO, Weslley. 16 estados têm ocupação de mais de 90\%, três não possuem mais vagas. CNN Brasil, São Paulo, 17 mar. 2021. Disponível em: https://www.cnnbrasil.com.br/saude/2021/03/17/16estados-estao-em-colapso-no-sistema-de-saude-tres-nao-tem-mais-vagas. Acesso em: 4 abr. 2021.

6 CANCIAN, Natália; SERAPIÃO, Fabio. País tem mais de mil cidades com dificuldades no abastecimento de oxigênio. Folha de S.Paulo, São Paulo, 7 abr. 2021. Disponível em: https://www1.folha.uol.com.br/ equilibrioesaude/2021/04/pais-tem-mais-de-mil-cidades-com-dificuldades-no-abastecimento-de-oxigenio. shtml. Acesso em: 4 abr. 2021.

7 CANZIAN, Fernando. SUS nos estados não tem leitos de UTI contra o coronavírus. Folha de S.Paulo, São Paulo, 15 mar. 2020. Disponível em: https://www1.folha.uol.com.br/equilibrioesaude/2020/03/sus-nosestados-nao-tem-leitos-de-uti-contra-o-coronavirus.shtml?origin=uol. Acesso em: 4 abr. 2021.

8 TIENGO, Rodolfo. Diante de colapso, Ribeirão Preto não tem condições de abrir mais UTIs para Covid, diz Prefeitura: ‘Estamos no limite’. G1, Ribeirão Preto e Franca, 1ํabr. 2021. Disponível em: https://g1.globo. $\mathrm{com} / \mathrm{sp} /$ ribeirao-preto-franca/noticia/2021/04/01/diante-de-colapso-ribeirao-preto-nao-tem-condicoes-deabrir-mais-utis-para-covid-diz-prefeitura-estamos-no-limite.ghtml. Acesso em: 4 abr. 2021.

9 'AMEAÇA à saúde pública global': colapso dos hospitais no Brasil é destaque na mídia estrangeira. $B B C$ News Brasil, 27 mar. 2021. Disponivel em: https://www.bbc.com/portuguese/brasil-56551811. Acesso em: 4 abr. 2021.

10 SOCIEDAD ESPAÑOLA DE MEDICINA INTENSIVA - CRÍTICA Y UNIDADES CORONARIAS (SEMICYUC). Recomendaciones éticas para la toma de decisiones en la situación excepcional de crisis por pandemia Covid-19 en las unidades de cuidados intensivos. Madrid: SEMICYUC, 16 mar. 2020. Disponível em: https://semicyuc.org/wp-content/uploads/2020/03/\%C3\%89tica_SEMICYUC-COVID-19.pdf. Acesso em: 4 abr. 2021. 
Como expressa o Boletim ํo 2/2021, do Comitê Extraordinário de Monitoramento Covid-19 da Associação Médica Brasileira, datado de 23.3.2021:

A falta de vagas em UTIs e o aumento anormal da demanda por vagas de leitos fazem urgentes também a adoção de critérios técnicos-científicos de triagem dos pacientes que devem ocupar os leitos disponíveis, com fundamentos éticos em defesa das vidas dos brasileiros, com diretrizes para todos os médicos do País; A escassez de recursos humanos, de insumos farmacológicos e de material de apoio transcende a simplicidade da utilização dos centros cirúrgicos como terapia intensiva. A transformação da sala de cirurgia em UTI deve ser pontual. É uma exceção, não regra. ${ }^{11}$

A seguir, procura-se identificar elementos jurídicos e parâmetros úteis para estabelecer a alocação dos recursos escassos, conforme escopo deste estudo.

\section{Avaliação jurídica}

\subsection{Legislação aplicável}

Inicialmente, sublinha-se que não foi localizada uma lei específica que estabeleça procedimentos e parâmetros que possam lastrear a decisão sobre a priorização de pacientes. ${ }^{12}$ A Lei $n$ ㅇ $13.979 / 2020$, que dispõe sobre as medidas para enfrentamento da emergência de saúde pública de importância internacional decorrente do coronavírus responsável pelo surto de 2019, não aprofundou balizas para alocação de recursos.

Apesar do amplo arcabouço legislativo, não há parâmetros nas leis que permitam chegar a conclusões definitivas para o cenário em análise. 0 Estatuto da Criança e do Adolescente (Lei no 8.069/1990) determina a priorização de quem

11 ASSOCIAÇÃO MÉDICA BRASILEIRA. Boletim 02/2021. Comitê Extraordinário de Monitoramento Covid-19 (CEM COVID_AMB). 23 mar. 2021. Disponível em: https://www.amib.org.br/fileadmin/user_upload/ amib/2021/marco/23/Boletim-CEM_COVID_AMB__2-_V.pdf. Acesso em: 4 abr. 2021.

12 Com idêntica conclusão, e interessante leitura sobre o tema, recomenda-se também o texto: MARMELSTEIN, George; MOROZOWSKI, Ana Carolina. Que vidas salvar? Escassez de leitos de UTI, critérios objetivos de triagem e a pandemia da Covid-19. Revista Publicum, Rio de Janeiro, v. 6, n. 1, p. 94-117, 2020. 0 texto oferece também importante advertência sobre a incompatibilidade de rediscussão da aplicação dos critérios na esfera judicial, inclusive em virtude da existência de outros pacientes, do caráter dinâmico dos quadros clínicos e do aspecto estritamente das ciências da saúde que devem pautar as decisões de triagem. 
não atingiu a maioridade, porém de modo análogo ocorre com o Estatuto do Idoso (Lei no 10.741/2003). ${ }^{13}$

A teor do Estatuto da Criança e do Adolescente:

Art. 4ํ É dever da família, da comunidade, da sociedade em geral e do poder público assegurar, com absoluta prioridade, a efetivação dos direitos referentes à vida, à saúde, à alimentação, à educação, ao esporte, ao lazer, à profissionalização, à cultura, à dignidade, ao respeito, à liberdade e à convivência familiar e comunitária. ${ }^{14}$

Parágrafo único. A garantia de prioridade compreende:

a) primazia de receber proteção e socorro em quaisquer circunstâncias;

b) precedência de atendimento nos serviços públicos ou de relevância pública;

c) preferência na formulação e na execução das políticas sociais públicas;

d) destinação privilegiada de recursos públicos nas áreas relacionadas com a proteção à infância e à juventude.

A Constituição reforça a priorização e inclui os "jovens":

Art. 227. É dever da família, da sociedade e do Estado assegurar à criança, ao adolescente e ao jovem, com absoluta prioridade, o direito à vida, à saúde, à alimentação, à educação, ao lazer, à profissionalização, à cultura, à dignidade, ao respeito, à liberdade e à convivência familiar e comunitária, além de colocá-los a salvo de toda forma de negligência, discriminação, exploração, violência, crueldade e opressão.

De acordo com o Estatuto do Idoso, art. 15, §7ํㅡ, “Em todo atendimento de saúde, os maiores de oitenta anos terão preferência especial sobre os demais idosos, exceto em caso de emergência". Em nossa análise, essa previsão não

13 Observa-se que “a Constituição Federal incorporou no ordenamento jurídico brasileiro a denominada doutrina da proteção integral, traduzida no princípio do melhor interesse da criança e do adolescente, o que the confere prioridades inclusive na área da saúde" (TEIXEIRA, Ana Carolina Brochado; PEREIRA, Paula Moura Francesconi de Lemos. A participação de crianças e adolescentes em ensaios clínicos: uma reflexão baseada nos princípios do melhor interesse, solidariedade e autonomia. Revista Brasileira de Direito Civil, Rio de Janeiro, v. 9, jul./set. 2016).

14 Cf. BARBOZA, Heloisa Helena. Bioética e biodireito: quem defende os interesses da criança? In: SCHRAM, F. R.; BRAZ, M. (Org.). Bioética e saúde: novos tempos para mulheres e crianças? Rio de Janeiro: Editora Fiocruz, 2005 (Criança, mulher e saúde, p. 125-138). 
permite concluir pela preferência para ocupação de leitos hospitalares. Além disso, dispõe o referido Estatuto:

Art. 3ํ É obrigação da família, da comunidade, da sociedade e do Poder Público assegurar ao idoso, com absoluta prioridade, a efetivação do direito à vida, à saúde, à alimentação, à educação, à cultura, ao esporte, ao lazer, ao trabalho, à cidadania, à liberdade, à dignidade, ao respeito e à convivência familiar e comunitária.

Na Lei Orgânica da Saúde (Lei do SUS), Lei no 8.080/1990, não há balizas especiais de priorização extraíveis para o tema sob exame. Igualmente, constata-se a omissão do Ministério da Saúde em estabelecer parâmetros sobre a alocação de recursos escassos. ${ }^{15}$ Diante do vácuo, as guidelines a serem consideradas devem ser verificadas em entidades de classe, ainda que tais instrumentos não sejam ideais. No que tange às normas do Conselho Federal de Medicina, vale sublinhar:

- Resolução CFM no 2.156/2016 estabelece os critérios de admissão e alta em Unidade de Terapia Intensiva;

- Recomendação CFM nำ 1/2015 sobre Comitês de Bioética;

- Código de Ética Médica - Resolução CFM no 2.217/2018;

- Resolução CFM № 1.805/2006 - Sobre cuidados paliativos.

Salienta-se também que a Associação Brasileira de Medicina Intensiva editou em 2020 os Princípios de triagem em situações de catástrofes e as particularidades da Pandemia Covid-19. Além disso, o emprego de algoritmos pode ser considerado. ${ }^{16}$ Há iniciativas nacionais ${ }^{17}$ neste sentido, contudo, em caso de desenvolvimento de uma ferramenta própria, é preciso lembrar que as "máquinas" vão atuar segundo os critérios que recebam. Portanto, será preciso primeiro

15 WANG, Daniel Wei Liang; LUCCA-SILVEIRA, Marcos de. Como na Itália, falta de UTI nos fará escolher entre quem vive e quem morre. Folha de S.Paulo, 1 abr. 2020. Disponível em: https://www1.folha.uol.com.br/ ilustrissima/2020/04/como-na-italia-falta-de-uti-nos-fara-escolher-entre-quem-vive-e-quem-morre.shtml. Acesso em: 4 abr. 2021.

16 O uso de ferramentas automatizadas exige a revisão constante, em vista das novas descobertas. Como exemplifica Rosenbaum, "Se decidíssemos não intubar pacientes com Covid-19 por mais de 10 dias, mas depois soubéssemos que esses pacientes precisam de 15 dias para se recuperar, precisariamos alterar nossos algoritmos" (ROSENBAUM, L. Facing Covid-19 in Italy - Ethics, logistics, and therapeutics on the epidemic's front line. New England Journal of Medicine, v. 382, n. 21, p. 1873-1875, 2020).

17 COLLUCCI, Cláudia. Algoritmos e inteligência artificial podem ajudar Brasil a decidir sobre leitos de UTI. Folha de S.Paulo, São Paulo, 24 mar. 2020. Disponível em: https://www1.folha.uol.com.br/equilibrio esaude/2020/03/algoritmos-e-inteligencia-artificial-podem-ajudar-brasil-a-decidir-sobre-leitos-de-uti. shtml?origin=uol. Acesso em: 4 abr. 2021. 
estabelecer prioridades para depois alimentar os sistemas que tomarão as decisões difíceis segundo os parâmetros que receberam.

\subsection{Escolhas dificeis, porém, necessárias. Considerações preliminares}

Recomenda-se que a definição dos critérios seja compartilhada, sobretudo com a definição de um plano de triagem de maneira a evitar decisões individuais. A análise deve levar em conta os recursos do sistema como um todo (não apenas de um hospital tomado de modo isolado). É igualmente de central importância a interlocução com os outros dispositivos de saúde do sistema, sobretudo por meio de centrais de regulação.

0 distanciamento da equipe de triagem da equipe médica é uma estratégia importante para assegurar decisões dentro de parâmetros éticos, ${ }^{18}$ inclusive para evitar viés, de maneira que se promove um cegamento em relação a aspectos como elementos raciais ou faixas de renda. "In addition to removing the responsibility for triage decisions from the bedside clinicians, committee members should also take on the task of communicating the decision to the family". ${ }^{19}$

A oferta e demanda das vagas em UTI devem ser reavaliadas de maneira constante. No difícil equilíbrio imposto pela escassez de recursos, o excesso de priorização (admissão de pacientes não prioritários) ou a subtriagem (subutilizando-se dos recursos) distanciam-se da busca pela escalabilidade e maximização do uso dos recursos e por salvar mais vidas. ${ }^{20}$ Ademais, devem-se manter atualizadas as autoridades de saúde. Deve-se também avaliar a possibilidade de abertura de novos leitos e profissionais se tal possibilidade for factível. Da exposição de motivos da Resolução CFM no 2.156/2016, consta ainda a seguinte orientação que não é imperativa, mas que deve ser levada em conta: "Recomenda-se que o número de leitos de UTI em um hospital seja proporcional ao número total de leitos da instituição e ao seu perfil de atendimento, variando em torno de $10 \%$ a $25 \%$ no Brasil".

18 SATOMI, Erika et al. Alocação justa de recursos de saúde escassos diante da pandemia de Covid-19: considerações éticas. Einstein (São Paulo), São Paulo, v. 18, 2020. Disponível em: https://journal.einstein.br/ pt-br/article/alocacao-justa-de-recursos-de-saude-escassos-diante-da-pandemia-de-covid-19-consideracoeseticas/. Acesso em: 4 abr. 2021.

19 TRUOG, Robert D.; MITCHELL, Christine; DALEY, George Q. The toughest triage - Allocating ventilators in a pandemic. New England Journal of Medicine, v. 382, n. 21, p. 1973-1975, 23 mar. 2020. Disponível em: https://www.nejm.org/doi/full/10.1056/nejmp2005689. Acesso em: 4 abr. 2021.

20 LECLERC, Thomas et al. Prioritisation of ICU treatments for critically ill patients in a Covid-19 pandemic with scarce resources. Anaesthesia, Critical Care \& Pain Medicine, v. 39, n. 3. p. 333-339, 17 maio 2020. 
Em relação aos profissionais responsáveis por triagem, é recomendada a existência de suporte inclusive por equipes de saúde mental, dada a elevada carga de responsabilidade e desgaste e alto nível de estresse associados a esta atividade. $^{21}$

Vale destacar que o objetivo dos protocolos de triagem não é exclusão de certos pacientes, por exemplo, por apresentarem determinada doença. A finalidade de um protocolo de triagem é maximizar o uso de recursos de cuidados intensivos para pacientes, identificar previamente os critérios, melhorando os fluxos e empregando da melhor maneira os recursos disponíveis.

\subsection{Parâmetros identificados}

A Resolução CFM no 2.156/2016 define níveis de prioridade para internação que devem ser observados nas escolhas de alocações de leitos. A teor da resolução, observa-se:

Art. 1ำ As admissões em unidade de tratamento intensivo (UTI) devem ser baseadas em: diagnóstico e necessidade do paciente; serviços médicos disponíveis na instituição; priorização de acordo com a condição do paciente; disponibilidade de leitos; potencial benefício para o paciente com as intervenções terapêuticas e prognóstico.

Em relação às prioridades, a Resolução CFM no 2.156/2016 regulamenta a priorização da admissão em unidade de tratamento intensivo e estabelece uma escala que coloca como primeira prioridade os pacientes que "necessitam de intervenções de suporte à vida, com alta probabilidade de recuperação e sem nenhuma limitação de suporte terapêutico" e como nível mais baixo (Prioridade 5), os "Pacientes com doença em fase de terminalidade, ou moribundos, sem possibilidade de recuperação".

21 "The angst that clinicians may experience when asked to withdraw ventilators for reasons not related to the welfare of their patients should not be underestimated - it may lead to debilitating and disabling distress for some clinicians. One strategy for avoiding this tragic outcome is to use a triage committee to buffer clinicians from this potential harm. We believe that such a committee should be composed of volunteers who are respected clinicians and leaders among their peers and the medical Community" (TRUOG, Robert D.; MITCHELL, Christine; DALEY, George Q. The toughest triage - Allocating ventilators in a pandemic. New England Journal of Medicine, v. 382, n. 21, p. 1973-1975, 23 mar. 2020. Disponivel em: https://www.nejm. org/doi/full/10.1056/nejmp2005689. Acesso em: 4 abr. 2021; SATOMI, Erika et al. Alocação justa de recursos de saúde escassos diante da pandemia de Covid-19: considerações éticas. Einstein (São Paulo), São Paulo, v. 18, 2020. Disponível em: https://journal.einstein.br/pt-br/article/alocacao-justa-de-recursosde-saude-escassos-diante-da-pandemia-de-covid-19-consideracoes-eticas/. Acesso em: 4 abr. 2021). 
Nessa linha, a Resolução CFM no 2.156/2016 assim estabelece:

Art. 6 A priorização de admissão na unidade de tratamento intensivo (UTI) deve respeitar os seguintes critérios: $\S$ 1ํ- Prioridade 1: Pacientes que necessitam de intervenções de suporte à vida, com alta probabilidade de recuperação e sem nenhuma limitação de suporte terapêutico $\S 20$ - Prioridade 2: Pacientes que necessitam de monitorização intensiva, pelo alto risco de precisarem de intervenção imediata, e sem nenhuma limitação de suporte terapêutico. $\S 3$ 으 Prioridade 3: Pacientes que necessitam de intervenções de suporte à vida, com baixa probabilidade de recuperação ou com limitação de intervenção terapêutica. $\S 4^{\circ}$ - Prioridade 4: Pacientes que necessitam de monitorização intensiva, pelo alto risco de precisarem de intervenção imediata, mas com limitação de intervenção terapêutica. $\S$ 5 - Prioridade 5: Pacientes com doença em fase de terminalidade, ou moribundos, sem possibilidade de recuperação. Em geral, esses pacientes não são apropriados para admissão na UTI (exceto se forem potenciais doadores de órgãos). No entanto, seu ingresso pode ser justificado em caráter excepcional, considerando as peculiaridades do caso e condicionado ao critério do médico intensivista.

Uma leitura próxima é identificada na análise de Maves e outros, ao listarem situações em que a internação não seria adequada:

In a broad sense, patients who present for tertiary triage are going to fit into one of three categories: (1) too well to benefit from critical care, (2) too sick to benefit from critical care because of severe underlying illness or a poor likelihood of surviving their hospitalization, or (3) sick enough to benefit from critical care. ${ }^{22}$

Estes elementos também são bastante próximos das hipóteses indicadas pela Sociedad Española de Medicina Intensiva, Crítica y Unidades Coronarias, ${ }^{23}$ como modelo decisório consagrado:

22 MAVES, Ryan C. et al. Triage of scarce critical care resources in Covid-19 an implementation guide for regional allocation an expert panel report of the Task Force for Mass Critical Care and the American College of Chest Physicians. CHEST, Elsevier, v. 158, p. 212-225, jul. 2020. Disponivel em: https://journal. chestnet.org/article/S0012-3692(20)30691-7/fulltext. Acesso em: 4 abr. 2021.

23 SOCIEDAD ESPAÑOLA DE MEDICINA INTENSIVA, CRÍTICA Y UNIDADES CORONARIAS (SEMICYUC). Recomendaciones éticas para la toma de decisiones en la situación excepcional de crisis por pandemia Covid-19 en las unidades de cuidados intensivos. Madrid: SEMICYUC, 16 mar. 2020. Disponível em: https://semicyuc.org/wp-content/uploads/2020/03/\%C3\%89tica_SEMICYUC-COVID-19.pdf. Acesso em: 4 abr. 2021. 


\section{Pacientes con Prioridad 1}

Serán pacientes críticos e inestables. Necesitan monitorización y tratamiento intensive que no puede ser proporcionado fuera de la $\mathrm{UCl}$ (ventilación mecánica invasiva, depuración renal continua...

Pacientes con Prioridad 2

Pacientes que precisan monitorización intensiva y pueden necesitar intervenciones inmediatas. Son pacientes que no estarán ventilados de forma invasiva, pero con altos requerimientos de oxigenoterapia con PaO2/FiO2 menor de 200 o menor de 300 con fracaso de otro órgano.

Pacientes con Prioridad 3

Se trata de pacientes inestables y críticos que tienen pocas posibilidades de recuperarse a causa de su enfermedad de base o de la aguda. Pueden recibir tratamiento intensivo para aliviar su enfermedad aguda, pero también establecerse límites terapéuticos como, por ejemplo, no intubar y/o no intentar la reanimación cardiopulmonar.

Pacientes con Prioridad 4

Pacientes cuyo ingreso no está generalmente indicado debido a un beneficio mínimo o improbable por enfermedad de bajo riesgo. Pacientes cuya enfermedad terminal e irreversible hace inminente su muerte.

Em cenário de crise, aponta a Sociedad Española de Medicina Intensiva, Crítica y Unidades Coronarias que os pacientes nas prioridades 3 e 4 não devem ser encaminhados a unidades de tratamento intensivo. ${ }^{24}$ Priorizam-se os Pacientes Prioridade 1 e, somente em seguida, Pacientes Prioridade 2.

Outra decisão difícil diz respeito à antecipação da alta da UTI de pacientes em virtude da falta de recursos. A chamada triagem reversa (reverse trial) é uma medida importante, mas sua adoção não pode ser feita de maneira simplista. Recomenda-se a constante reavaliação do quadro de pacientes internados, assim como a integração da alocação de recursos em UTI com leitos hospitalares mais simples. Ademais, tal como os consensos sobre a internação em UTI, as hipóteses de alta devem ser estabelecidas previamente. ${ }^{25}$

24 SOCIEDAD ESPAÑOLA DE MEDICINA INTENSIVA, CRÍTICA Y UNIDADES CORONARIAS (SEMICYUC). Recomendaciones éticas para la toma de decisiones en la situación excepcional de crisis por pandemia Covid-19 en las unidades de cuidados intensivos. Madrid: SEMICYUC, 16 mar. 2020. Disponivel em: https://semicyuc.org/wp-content/uploads/2020/03/\%C3\%89tica_SEMICYUC-COVID-19.pdf. Acesso em: 4 abr. 2021.

25 EINAV, Sharon et al. Surge capacity logistics: care of the critically ill and injured during pandemics and disasters: CHEST consensus statement. CHEST, n. 146, p. e17S-43S, 2014. 
Retomando-se a Resolução CFM no 2.156/2016, é importante também destacar que os pacientes classificados como Prioridade 2 ou 4 devem prioritariamente ser admitidos em unidades de cuidados intermediários (semi-intensivas) (Resolução CFM ํo 2.156/2016, art. 7ㅇ). A triagem de leitos deve, portanto, seguir a Resolução CFM no 2.156/2016. Esta norma também estabelece critérios de alta. De acordo com o art. 10, são hipóteses para alta das unidades de tratamento intensivo (UTI):

a) Paciente que tenha seu quadro clínico controlado e estabilizado;

b) Paciente para o qual tenha se esgotado todo o arsenal terapêutico curativo/restaurativo e que possa permanecer no ambiente hospitalar fora da UTI de maneira digna e, se possível, junto com sua família.

Dessa maneira, embora seja frequente a admissão na UTI de pacientes em condições muito graves e com baixa perspectiva de benefício, é preciso observar se há efetivo potencial no suporte, evitando-se a obstinação terapêutica. ${ }^{26}$ Nesse sentido, estabelece a Resolução CFM no 1.805/2006:

Na fase terminal de enfermidades graves e incuráveis é permitido ao médico limitar ou suspender procedimentos e tratamentos que prolonguem a vida do doente, garantindo-lhe os cuidados necessários para aliviar os sintomas que levam ao sofrimento, na perspectiva de uma assistência integral, respeitada a vontade do paciente ou de seu representante legal.

Vale salientar que, a teor da Resolução CFM no 1.805/2006, art. 1ㅇ:

É permitido ao médico limitar ou suspender procedimentos e tratamentos que prolonguem a vida do doente em fase terminal, de enfermidade grave e incurável, respeitada a vontade da pessoa ou de seu representante legal.

Deve-se observar ainda a autonomia do paciente, inclusive a possibilidade de recusa de tratamentos. ${ }^{27}$

26 TEPEDINO, Gustavo; SCHREIBER, Anderson. O extremo da vida - Eutanásia, accanimento terapêutico e dignidade humana. Revista Trimestral de Direito Civil, Rio de Janeiro, v. 39, jul./set. 2009.

27 Sobre esta interface há farta literatura, recomenda-se: DWORKIN, Gerald. The theory and practice of autonomy. Cambridge: Cambridge University Press, 2008; BARROSO, Luís Roberto. Legitimidade da recusa de transfusão de sangue por testemunhas de Jeová. Dignidade humana, liberdade religiosa e escolhas existenciais. In: CARBONELL, Miguel et al. Direitos, deveres e garantias fundamentais. Salvador: 
A despeito de se identificar balizas, observa-se que no contexto de uma crise sanitária mais aguda, e do grau de colapso do sistema, as regras desenhadas podem não dar conta. Por exemplo, não há critérios para desempate entre pacientes do mesmo nível de prioridade. Nesse cenário, pode ser necessário aprofundar as orientações apontadas pelo CRM. A partir de uma análise do direito brasileiro, das normas legais e deontológicas, recomendam-se como parâmetros úteis:

- não há parâmetros isolados que sejam suficientes para decidir sobre alocação de recursos em UTI;28

- é preciso estabelecer medidas para evitar vieses (bias) ou medidas discriminatórias;

- não constituem elementos adequados para decisão a consideração da profissão, família, nível de escolaridade;

- a definição de balizas deve ser feita por uma comissão e não por uma única pessoa; recomenda-se também o monitoramento de tais decisões;

- a separação entre a triagem/alocação de recursos e atendimento potencializa decisões eticamente adequadas;

- "não prioridade por doença: durante uma pandemia, pacientes portadores de doenças outras, que não a doença pandêmica, devem ser triados pelos mesmos critérios de prioridade de atendimento";29

- há um forte componente emocional na alocação, o que recomenda suporte psicológico à equipe de atenção à saúde e presença de profissionais com maior experiência;

- oferta da melhor atenção disponível aos pacientes que não possam receber atenção pela internação em UTI, ${ }^{30}$ inclusive cuidados paliativos;

JusPodivm, 2011. p. 661-707; O'NEILL, Onora. Paternalism and partial autonomy. Journal of Medical Ethics, v. 10, p. 173-178, 1984; BARBOZA, Heloisa Helena Gomes. Vulnerabilidade e cuidado: aspectos jurídicos. In: PEREIRA, Tânia da Silva; OLIVEIRA, Guilherme de (Org.). Cuidado \& vulnerabilidade. São Paulo: Atlas, 2009. p. 106-118; TEIXEIRA, Ana Carolina Brochado. Saúde, corpo e autonomia privada. Rio de Janeiro: Renovar, 2010; BUCHANAN, Allen; BROCK, Dan. Deciding for others: the ethics of surrogate decision making. Cambridge: Cambridge University Press, 1989; FEINBERG, Joel. Legal paternalism. Canadian Journal of Philosophy, v. I, n. 1, p. 105-124, set. 1971.

28 "No single value is sufficient alone to determine which patients should receive scarce resources" (EMANUEL, Ezekiel J. et al. Fair allocation of scarce medical resources in the time of Covid-19. New England Journal of Medicine, v. 382, n. 21, p. 2049-2055, 2020. Disponivel em: https://www.nejm.org/ doi/full/10.1056/nejmsb2005114. Acesso em: 4 abr. 2021).

29 SATOMI, Erika et al. Alocação justa de recursos de saúde escassos diante da pandemia de Covid-19: considerações éticas. Einstein (São Paulo), São Paulo, v. 18, 2020. Disponível em: https://journal. einstein.br/pt-br/article/alocacao-justa-de-recursos-de-saude-escassos-diante-da-pandemia-de-covid-19consideracoes-eticas/. Acesso em: 4 abr. 2021.

3o MAVES, Ryan C. et al. Triage of scarce critical care resources in Covid-19 an implementation guide for regional allocation an expert panel report of the Task Force for Mass Critical Care and the American College of Chest Physicians. CHEST, Elsevier, v. 158, p. 212-225, jul. 2020. Disponivel em: https://journal. chestnet.org/article/S0012-3692(20)30691-7/fulltext. Acesso em: 4 abr. 2021. 
- a transformação de espaços deve ser vista como medida excepcional. ${ }^{31}$

Mostra-se bastante interessante também a confrontação dos critérios que são levantados pela Sociedad Española de Medicina Intensiva, Crítica y Unidades Coronarias, que incluem: o contexto de crise sanitária não permite a desconsideração aos casos incuráveis, de maneira que devem ser observados os cuidados paliativos, $^{32}$ em harmonia com a Resolução CFM nำ1.805/2006, art. 2ㅇ․ ${ }^{33}$

No estado do Rio Grande do Sul, foi publicado pelo Conselho Regional de Medicina do Estado do Rio Grande Do Sul - CREMERS, o Protocolo de Regulação para casos de Covid-19 para Unidades de Terapia Intensiva de Alta Complexidade, por meio da Resolução CREMERS nำ12/2020, sobretudo com vistas à regulação das transferências. ${ }^{34} \mathrm{O}$ protocolo destaca, em harmonia com o acima exposto:

- necessidade de priorizar o atendimento para pacientes com probabilidade diagnóstica de infecção por Covid-19, de acordo com as orientações dos órgãos governamentais;

31 “Em relação à infraestrutura física, deve-se observar a excepcionalidade da situação de emergência sanitária para criar e adaptar áreas inicialmente não planejadas para UTIs para a implantação dos LPCC-19, provendo-as de condições mínimas necessárias para a segurança dos pacientes e dos profissionais de saúde" (CONSELHO FEDERAL DE MEDICINA - CFM. Ofício CFM no 1749/2020 - GABIN. 20 mar. 2020. Disponível em: https://www.amib.org.br/fileadmin/user_upload/amib/2020/marco/21/Ofi_cio_CFM_ no_1749-2020-GABIN.pdf. Acesso em: 4 abr. 2021). o Asian Critical Care Clinical Trials Group oferece uma lista de hipóteses de adaptações recomendadas. Para um exemplo de fácil compreensão, quando um quarto individual não estiver disponível, a orientação é agrupar casos semelhantes em um quarto espaçados na UTI (PHUA, Jason et al. Intensive care management of coronavirus disease 2019 (Covid-19): challenges and recommendations. Lancet Respir Med, v. 5, n. 8, p. 506-517, maio 2020). Outras possibilidades de maximização de recursos estão disponíveis em: AZIZ, Shadman et al. Managing ICU surge during the Covid-19 crisis: rapid guidelines. Intensive Care Medicine, v. 46, n. 7, p. 1303-1325, 2020.

32 "Pacientes gravemente enfermos acometidos pela COVID-19 devem receber Cuidados Paliativos pois apresentam alto risco de morte com alta carga de sintomas. O tripé que sustenta a filosofia dos Cuidados Paliativos é: 0 respeito à autonomia do paciente, indissociável da boa comunicação; 0 controle adequado dos seus sintomas; 0 não prolongamento do morrer, diretamente associado: à adequada avaliação prognóstica e à garantia dos cuidados paliativos durante o morrer. As decisões quanto aos cuidados de fim de vida em situações de pandemias devem ser baseadas em prognóstico, valores e contexto" (ASSOCIAÇÃO DE MEDICINA INTENSIVA BRASILEIRA - AMIB. Recomendações para os cuidados paliativos em UTI nas situações de catástrofes (particularidades do Covid-19). Disponível em: https://www.amib. org.br/fileadmin/user_upload/amib/2021/marco/23/Recomendacao_Comite_de_Cuidados_Paliativos_ COVID_19_AMIB_2021.pdf. Acesso em: 4 abr. 2021). Nessa linha também: SICP - SOCIETÀ ITALIANA DI CURE PALLIATIVE. Comunicato stampa SICP - FCP sulle "Raccomandazioni di etica clinica per l'ammissione a trattamenti intensivi e per la loro sospensione, in condizioni eccezionali di squilibrio tra necessità e risorse disponibili" - SIAARTI. 6 marzo 2020. Disponível em: https://www.fedcp.org/images/file/1102/ cs-sicp-fcp10-mar-2020.pdf. Acesso em: 4 abr. 2021.

33 “o doente continuará a receber todos os cuidados necessários para aliviar os sintomas que levam ao sofrimento, assegurada a assistência integral, o conforto físico, psíquico, social e espiritual".

34 ENTIDADES DE FISCALIZAÇÃO DO EXERCÍCIO DAS PROFISSÕES LIBERAIS; CONSELHO REGIONAL DE MEDICINA DO ESTADO DO RIO GRANDE DO SUL. Resolução n. 12, de 27 de maio de 2020. Aprova o Protocolo de Regulação para casos de Covid-19 para Unidades de Terapia Intensiva de Alta Complexidade. Disponivel em: https://cremers.org.br/wp-content/uploads/2020/05/27.05.2020-Resolu\%C3\%A7\% C3\%A3o-122020-do-CREMERS.pdf. 
- existência de outros pacientes com necessidade de cuidados intensivos, não portadores de Covid-19, que continuarão existindo e demandando a atenção das equipes multidisciplinares de UTI;

- necessidade de destinar os recursos disponíveis para pacientes com uma probabilidade razoável de sobrevivência com qualidade de vida aceitável.

$\mathrm{Na}$ hipótese de esgotamento dos recursos para o atendimento de todas as pessoas que deles necessitem, são apontados como critérios de alocação dos recursos, com priorização de casos, as seguintes três hipóteses:

a) urgência médica: segundo este racional, o recurso é alocado ao caso mais grave, independentemente de sua chance estimada de recuperação;

b) utilidade: de acordo com este racional, o recurso é alocado ao caso com maior sobrevida estimada, independentemente de sua gravidade e de seu risco de óbito caso não recebesse aquele recurso;

c) beneficio: conforme este racional, sopesam-se urgência e utilidade, considerando-se a sobrevida da população de pessoas com indicação de receber o recurso (incluindo os casos que o receberão e aqueles que não o receberão), com o objetivo de produzir o maior impacto na sobrevida daquela população como um todo e não na dos indivíduos isoladamente. Assim, de acordo com esta lógica, priorizam-se os casos com a maior diferença entre as expectativas de vida caso recebam ou caso não recebam o recurso.

Diante das três hipóteses, a solução apontada pelo Conselho consiste no princípio do benefício. Se não possível adotar tais orientações, a resolução sugere como referências:

d) gravidade dos casos e risco de óbito sem a transferência;

e) complexidade de estrutura de saúde disponível no local em que os casos estão naquele momento;

f) características biológicas e clínicas dos casos que possam influenciar na probabilidade estimada de reversão do quadro agudo (como comorbidades preexistentes, entre outras).

\subsection{Diretrizes da Sociedad Española de Medicina Intensiva, Crítica y Unidades Coronarias}

A Sociedad Española de Medicina Intensiva, Crítica y Unidades Coronarias ${ }^{35}$ apresenta critérios gerais e específicos que se mostram bastante interessantes e

\footnotetext{
SOCIEDAD ESPAÑOLA DE MEDICINA INTENSIVA, CRITICA Y UNIDADES CORONARIAS (SEMICYUC). Recomendaciones éticas para la toma de decisiones en la situación excepcional de crisis por pandemia Covid-19 en las unidades de cuidados intensivos. Madrid: SEMICYUC, 16 mar. 2020. Disponível em: https://semicyuc. org/wp-content/uploads/2020/03/\%C3\%89tica_SEMICYUC-COVID-19.pdf. Acesso em: 4 abr. 2021.
} 
coerentes com as normas legais e deontológicas brasileiras. Ademais, revelam-se mais densos do que as normas nacionais.

Dada a extensão das orientações, a seguir foram indicados os elementos considerados mais úteis para complementar as considerações acima expostas e também à vista da legislação brasileira:

1. Sempre deve haver um grande benefício esperado e reversibilidade.

2. Diante de um paciente com indicação de ventilação mecânica, e sem disponibilidade de recursos ideais, avaliar a possibilidade de distribuir os pacientes entre as UTI disponíveis.

3. Em situações em que os recursos necessários excedem os disponíveis, devem ser aplicados critérios de triagem para admissão na UTI.

4. Aplicação de critérios rígidos de admissão à UTI com base na maximização do benefício do bem comum.

5. Incentivar os procedimentos que facilitem o desmame de ventilação mecânica de acordo com a medicina baseada em evidências e agilizar os circuitos de transferência para a planta e centros de referência, para liberar recursos para outros pacientes.

6. Em caso de complicações ou curso clínico ruim esperado como funcional, propor retirada terapêutica sem demora devido à futilidade e iniciar a aplicação de medidas paliativas.

7. Em uma situação de adequação terapêutica, retirada de medidas e/ou má evolução, é apropriado encaminhar o paciente para uma área de menor complexidade para estabelecer o plano de cuidados paliativos.

8. Consultar o serviço de cuidados paliativos para garantir a continuidade de cuidado de pacientes dos quais há limitação de tratamentos e aliviar o seu sofrimento, incluindo sedação paliativa nos casos em que é preciso.

9. O paciente deve ser avaliado globalmente e não apenas a doença de forma isolada.

10. Estabelecer, em todos os pacientes admitidos nas estações de tratamento, hospitalização (internado por Covid-19 ou outros motivos), planejar com antecedência e documentar no prontuário médico, com definição clara da existência ou não de indicação de ventilação mecânica e/ou admissão na UTI e comunicá-la ao restante da equipe e especialidades. $\mathrm{Na}$ admissão do paciente na UTI, estabelecer se ele é ou não contribuinte de medidas invasivas e RCP.

11. Deve ser sempre consultado se o paciente tiver vontade de avançar e seguir suas instruções, consultando os representantes designados no documento diretivo antecipado. 
12. Diante de pacientes similares, levar em conta, anos ajustados, qualidade de vida (AVAC) ou QALY (Quality-Adjusted Life Year). ${ }^{36}$

A entidade espanhola levanta critérios específicos sobre idade cuja adoção não nos parece recomendável, salvo se for possível tecnicamente realizar associação, segundo fundamentos de medicina baseada em evidências, como estratégia para estabelecer um critério clínico. Erika Satomi ${ }^{37}$ preconiza a importância do critério da fragilidade clínica, conforme Escala de Fragilidade Clínica (CSHA Clinical Frailty Scale) proposta por Rockwood, ${ }^{38} \mathrm{em}$ conjugação com a Sofa (Sequential Organ Failure Assessment Score). A proposta de Rockwok, vale lembrar, consiste em um conjunto amplo de elementos, desenvolvido ao longo de anos. Conforme síntese oferecida pelo próprio autor:

\section{Box 1: The CSHA Clinical Frailty Scale}

1 Very fit - robust, active, energetic, well motivated and fit; these people commonly exercise regularly and are in the most fit group for their age

2 Well - without active disease, but less fit than people in category 1

3 Well, with treated comorbid disease - disease symptoms are well controlled compared with those in category 4

4 Apparently vulnerable - although not frankly dependent, these people commonly complain of being "slowed up" or have disease symptoms

5 Mildly frail - with limited dependence on others for instrumental activities of daily living

6 Moderately frail - help is needed with both instrumental and non-instrumental activities of daily living

7 Severely frail - completely dependent on others for the activities of daily living, or terminally ill

Note: $\mathrm{CSHA}=$ Canadian Study of Health and Aging.

36 Trata-se de uma medida de saúde amplamente empregada para orientar a alocação de recursos de saúde. O QALY é utilizado por exemplo pelo US Panel on Cost-Effectiveness in Health and Medicine, nos Estados Unidos e o National Institute of Health and Clinical Excellence (NICE) na Inglaterra. Para uma didática exposição sobre o QALY, confira-se: WEINSTEIN Milton; TORRANCE, George; MCGUIRE, Alistair. QALYs: the basics. Value Health, v. 13, n. 8, dez. 2010.

37 SATOMI, Erika et al. Alocação justa de recursos de saúde escassos diante da pandemia de Covid-19: considerações éticas. Einstein (São Paulo), São Paulo, v. 18, 2020. Disponível em: https://journal. einstein.br/pt-br/article/alocacao-justa-de-recursos-de-saude-escassos-diante-da-pandemia-de-covid-19consideracoes-eticas/. Acesso em: 4 abr. 2021.

38 ROCKWOOD, K. et al. A global clinical measure of fitness and frailty in elderly people. CMAJ, v. 173, n. 5, p. 489-495, ago. 2005. Disponível em: https://www.cmaj.ca/content/173/5/489. Acesso em: 4 abr. 2021. 
Satomi, ${ }^{39}$ a partir de Rockwood, sintetiza os critérios do autor, na seguinte tabela:

Tabela 2. Alocação de recursos segundo escala de fragilidade clínica de Rockwood et al. ${ }^{(11)}$

\begin{tabular}{lcc}
\hline Grau de prioridade & Rockwood & Alocação \\
\hline Alta prioridade & $1-3$ & UTI/ semi \\
\hline Prioridade intermediária & $4-6$ & ICU trial \\
\hline Baixa prioridade & 7 & Enfermaria - Cuidados Paliativos \\
\hline
\end{tabular}

UTI: unidade de terapia intensiva; ICU trial: tentativa de tratamento em unidade de terapia intensiva.

Vale também reproduzir a seguinte advertência de Satomi:

\begin{abstract}
Nos casos em que é optado por uma tentativa de tratamento em UTI (ICU trial), recomenda-se que fique claro, desde o início da internação em UTI, que existe um tempo em que se é esperado que o paciente melhore, e, caso isso não aconteça, esse dispositivo será retirado por falha terapêutica. O Sequential Organ Failure Assessment (SOFA) parece ser um dos fatores prognósticos de mortalidade intra-hospitalar durante a pandemia (Tabela 3).
\end{abstract}

Emanuel, em estudo publicado no New England Journal of Medicine, ${ }^{40}$ elenca valores bioéticos a serem considerados na alocação de recursos escassos. Para melhor visualização, sintetizamos as diretrizes do autor nos seguintes aspectos:

1-) Maximizar benefício;

2-) Tratar de modo igual pacientes iguais, com seleção aleatória por chegada quando o prognóstico for semelhante;

3-) Priorizar trabalhadores da saúde envolvidos com atendimento de outros pacientes;

39 SATOMI, Erika et al. Alocação justa de recursos de saúde escassos diante da pandemia de Covid-19: considerações éticas. Einstein (São Paulo), São Paulo, v. 18, 2020. Disponível em: https://journal. einstein.br/pt-br/article/alocacao-justa-de-recursos-de-saude-escassos-diante-da-pandemia-de-covid-19consideracoes-eticas/. Acesso em: 4 abr. 2021.

40 EMANUEL, Ezekiel J. et al. Fair allocation of scarce medical resources in the time of Covid-19. New England Journal of Medicine, v. 382, n. 21, p. 2049-2055, 2020. Disponível em: https://www.nejm.org/doi/ full/10.1056/nejmsb2005114. Acesso em: 4 abr. 2021. 
4-) As diretrizes de priorização devem diferir por intervenção e devem responder às mudanças nas evidências científicas;

5-) Pessoas que participam de pesquisas com vacinas devem ser priorizadas;

6-) Não deve haver diferença na alocação de recursos escassos entre pacientes com Covid-19 e aqueles com outras condições médicas.

Para melhor compreensão, transcrevemos os trechos mais relevantes do texto, ${ }^{41}$ em tradução livre para a língua portuguesa:

\section{Recomendação 1: Maximizar benefícios}

[...] Salvar mais vidas e mais anos de vida é um valor consensual em relatórios de especialistas

[...] qualquer equilíbrio entre vidas e anos de vida escolhido deve ser aplicado de forma consistente

[...] O tempo e as informações limitados durante uma emergência também desaconselham a incorporação da qualidade de vida futura dos pacientes e dos anos de vida ajustados pela qualidade na maximização dos benefícios.

[...] Como maximizar os benefícios é fundamental em uma pandemia, acreditamos que retirar um paciente de um ventilador ou leito de UTI para fornecê-lo a outros necessitados também é justificável e que os pacientes devem ser informados dessa possibilidade na admissão

Recomendação 2: Intervenções críticas da Covid-19 - teste, EPI, leitos de UTI, ventiladores, terapêutica e vacinas - devem ir primeiro para os profissionais de saúde da linha de frente e outros que cuidam de pacientes doentes e que mantêm a infraestrutura crítica em funcionamento, especialmente os trabalhadores que enfrentam um alto risco de infecção e cujo treinamento torna difícil substituí-los. [...].

Recomendação 3: Para pacientes com prognósticos semelhantes, a igualdade deve ser invocada e operacionalizada por meio de alocação aleatória, como uma loteria, em vez de um processo de alocação por ordem de chegada. O primeiro a chegar, primeiro a ser servido é usado para recursos como rins transplantáveis, onde a escassez é antiga e os pacientes podem sobreviver sem o recurso escasso [...].

41 EMANUEL, Ezekiel J. et al. Fair allocation of scarce medical resources in the time of Covid-19. New England Journal of Medicine, v. 382, n. 21, p. 2049-2055, 2020. Disponivel em: https://www.nejm.org/doi/ full/10.1056/nejmsb2005114. Acesso em: 4 abr. 2021. 
Recomendação 4: As diretrizes de priorização devem diferir por intervenção e devem responder às mudanças nas evidências científicas;

[...] Os resultados do Covid-19 foram significativamente piores em pessoas mais velhas e naqueles com condições crônicas. [...] "leitos de UTI e ventiladores são curativos ao invés de preventivos. Os pacientes que precisam deles enfrentam condições de risco de vida. A maximização dos benefícios requer a consideração do prognóstico - quanto tempo o paciente provavelmente viverá se tratado - o que pode significar dar prioridade a pacientes mais jovens e àqueles com menos doenças coexistentes. Isso é consistente com as diretrizes italianas [...]

Recomendação 5: Pessoas que participam de pesquisas para provar a segurança e eficácia de vacinas e terapêuticas devem receber alguma prioridade para as intervenções da Covid-19.

Recomendação 6: Não deve haver diferença na alocação de recursos escassos entre pacientes com Covid-19 e aqueles com outras condições médicas.

A legislação brasileira não trouxe uma regra que estabeleça prioridade de profissionais da saúde, o que coloca em questão a Recomendação 2, mas é preconizada também por autores brasileiros. ${ }^{42}$

A Amib (Associação de Medicina Intensiva Brasileira) e a Abramede (Associação Brasileira de Medicina de Emergência) estabeleceram o Protocolo de alocação de recursos em esgotamento durante a pandemia por Covid-19, o qual deve ser lido em conjunto com o presente parecer. Entre os critérios identificados, destacam-se:

1) Salvar vidas - análise por meio do escore Sofa (Sequential Organ Failure Assessment). Vale lembrar que, de acordo com este protocolo, quanto menor a pontuação, maior a prioridade. ${ }^{43}$

42 "O conceito que se defende é o de salvar vida de quem pode salvar mais vidas, pois, sem profissionais capacitados, todos os pacientes - e não apenas aqueles com COVID-19 - sofrerão maior risco de mortalidade e terão anos de vida perdidos" (SATOMI, Erika et al. Alocação justa de recursos de saúde escassos diante da pandemia de Covid-19: considerações éticas. Einstein (São Paulo), São Paulo, v. 18, 2020. Disponível em: https://journal.einstein.br/pt-br/article/alocacao-justa-de-recursos-de-saude-escassos-diante-da-pandemiade-covid-19-consideracoes-eticas/. Acesso em: 4 abr. 2021).

43 Este critério é também preconizado por outros autores no contexto do Covid (UNIVERSITY OF PITSBURG. Allocation of scarce critical care resources during a public health emergency. Executive summary. Department of Critical Care Medicine. 23 mar. 2020. Disponivel em: https://ccm.pitt.edu/sites/default/ files/Model\%20hospital\%20policy\%20for\%20allocation\%20of\%20critical\%20care_2020-03-23\%20web. pdf; SOCIEDAD ESPAÑOLA DE MEDICINA INTENSIVA, CRITICA Y UNIDADES CORONARIAS (SEMICYUC). Recomendaciones éticas para la toma de decisiones en la situación excepcional de crisis por pandemia 
2) Probabilidade de sobrevida - consideram-se a presença de comorbidades e a possibilidade de sobrevida.

3) Escala de performance funcional Ecog (Eastern Cooperative Oncologic Group) - capacidade funcional do paciente, por exemplo, aptidão ao autocuidado.

De maneira próxima, para Christian, "the key factors being considered when making disaster triage decisions need to include survival, quality of life, and the resources necessary to achieve these outcomes". ${ }^{44}$ Desta maneira, as decisões sobre alocação devem se pautar pela possibilidade de sobrevida, capacidade funcional e recursos necessários.

\subsection{Estruturação do sistema de triagem}

Maves $^{45}$ e outros propõem um conjunto de etapas para estabelecer o sistema de triagem. Em tradução livre, as etapas são as seguintes:

1. Inventário de recursos potenciais de UTI para um aumento na demanda; 2. Estabelecer marcos de identificação e início da triagem: conforme a demanda clínica atinge o estágio de crise e que os padrões de crise de atendimento, incluindo a triagem, devem ser iniciados; 3. Preparação do sistema de triagem.

Covid-19 en las unidades de cuidados intensivos. Madrid: SEMICYUC, 16 mar. 2020. Disponível em: https://semicyuc.org/wp-content/uploads/2020/03/\%C3\%89tica_SEMICYUC-COVID-19.pdf. Acesso em: 4 abr. 2021; SATOMI, Erika et al. Alocação justa de recursos de saúde escassos diante da pandemia de Covid-19: considerações éticas. Einstein (São Paulo), São Paulo, v. 18, 2020. Disponível em: https:// journal.einstein. br/pt-br/article/alocacao-justa-de-recursos-de-saude-escassos-diante-da-pandemiade-covid-19-consideracoes-eticas/. Acesso em: 4 abr. 2021). Em evento do Cremesp, registrou-se: “É igualmente importante ter um escore, como o Sequential Organ Failure Assessment (SOFA), tanto na admissão como na evolução do quadro do paciente, para a tomada de decisão. Também é relevante observar a funcionalidade do paciente na chegada e na evolução do quadro. No HC de Campinas foi utilizada a Escala de Funcionalidade de Karnofsky (KPS) - classicamente aplicada em pacientes oncológicos - na qual se observou uma forte associação com a evolução para limitação de suporte vital. Outra que forneceu dados interessantes foi a Escala de Funcionalidade de Katz, indicando que, quanto maior a pontuação, maior era o risco de o paciente vir a falecer. No protocolo do HC de Campinas foi utilizada, ainda, a Escala de Funcionalidade de PPS (Paliative Performance Scale), para a tomada de decisão sobre a alocação dos pacientes nas alas hospitalares" (CREMESP. Cremesp promove live sobre alocação de recursos escassos durante a pandemia da Covid-19. 3 ago. 2020. Disponivel em: http://covid-19.cremesp.org.br/?p=1088).

44 CHRISTIAN, Michael D. Triage. Critical Care Clinics, v. 35, n. 4, p. 575-589, 2019.

45 MAVES, Ryan C. et al. Triage of scarce critical care resources in Covid-19 an implementation guide for regional allocation an expert panel report of the Task Force for Mass Critical Care and the American College of Chest Physicians. CHEST, Elsevier, v. 158, p. 212-225, jul. 2020. Disponivel em: https://journal. chestnet.org/article/S0012-3692(20)30691-7/fulltext. Acesso em: 4 abr. 2021. 
Embora não estabeleça critérios, a proposta se mostra interessante na organização do sistema: ${ }^{46}$

\begin{tabular}{|c|c|}
\hline Ações específicas sugeridas & Responsáveis \\
\hline $\begin{array}{l}\text { 1. Inventário de recursos potenciais de UTI para } \\
\text { um aumento na demanda: } \\
\text { a. ventiladores físicos e camas (por exemplo, } \\
\text { sala de cirurgia, SRPA); } \\
\text { b. recursos humanos (equipe com treinamento } \\
\text { em UTI); } \\
\text { c. suprimentos e espaço para prestar cuidados } \\
\text { (por exemplo, medicamentos, itens descartá- } \\
\text { veis, EPI, SRPA). }\end{array}$ & Unidades de saúde individuais \\
\hline $\begin{array}{l}\text { 2. Estabelecimento de marcos de identificação } \\
\text { e início da triagem: conforme a demanda clínica } \\
\text { atinge o estágio de crise e os padrões de crise } \\
\text { de atendimento, incluindo a triagem, devem ser } \\
\text { iniciados: } \\
\text { a. a decisão de iniciar a triagem deve ser feita } \\
\text { por uma autoridade regional identificada com } \\
\text { consciência situacional das demandas regio- } \\
\text { nais de cuidados de saúde; } \\
\text { b. a triagem deve ser aplicada de forma consisten- } \\
\text { te em toda a região, com justificativa documenta- } \\
\text { da e supervisão da autoridade regional relevante. }\end{array}$ & $\begin{array}{l}\text { - Autoridades regionais de } \\
\text { saúde do governo (condado/ } \\
\text { estado/província/nacional). } \\
\text { - Autoridades regionais ou } \\
\text { nacionais de gestão de emer- } \\
\text { gências (por exemplo, CDC ou } \\
\text { equivalente, departamento de } \\
\text { saúde pública estadual/pro- } \\
\text { vincial). }\end{array}$ \\
\hline $\begin{array}{l}\text { 3. Preparação do sistema de triagem: } \\
\text { a. criar comitê central de triagem para a região, } \\
\text { com a função de coordenação e padronização. } \\
\text { Isso deve incluir a representação das principais } \\
\text { partes interessadas (médicos, enfermagem, } \\
\text { ética, legislação, pacientes e representantes da } \\
\text { comunidade); } \\
\text { b. identificar membros de equipes de triagem } \\
\text { terciária institucional e estruturas de apoio; } \\
\text { c. preparar e distribuir materiais de treinamento } \\
\text { para funcionários locais para padronização da } \\
\text { implementação. }\end{array}$ & $\begin{array}{l}\text { - Departamento de saúde pú- } \\
\text { blica/ministério da saúde. } \\
\text { - Hospitais locais com uma } \\
\text { UTI. }\end{array}$ \\
\hline
\end{tabular}

46 Para outro modelo, cf. EINAV, Sharon et al. Surge capacity logistics: care of the critically ill and injured during pandemics and disasters: CHEST consensus statement. CHEST, n. 146, p. e17S-43S, 2014. 


\subsection{Critérios não recomendados}

A idade não deve ser um critério excludente para internação. Nessa linha, em revisão das Recomendações Amib/Abramede de alocação de recursos em esgotamento durante a pandemia por Covid-19, ${ }^{47}$ consta a "A retirada deste critério e a inclusão de uma medida de funcionalidade em seu lugar é a principal diferença entre as duas versões, além, claro, do maior refinamento quanto a descrição das bases legais que amparam o protocolo".$^{48} \mathrm{Na}$ forma do Estatuto do Idoso:

Art. $2^{\circ} 0$ idoso goza de todos os direitos fundamentais inerentes à pessoa humana, sem prejuízo da proteção integral de que trata esta Lei, assegurando-se-Ihe, por lei ou por outros meios, todas as oportunidades e facilidades, para preservação de sua saúde física e mental e seu aperfeiçoamento moral, intelectual, espiritual e social, em condições de liberdade e dignidade.

O critério etário por si não se mostra adequado à legislação brasileira, que estabelece em inúmeras disposições a proteção do idoso de maneira prioritária. ${ }^{49}$

47 ASSOCIAC̄̃O DE MEDICINA INTENSIVA BRASILEIRA - AMIB; ASSOCIACCÃO BRASILEIRA DE MEDICINA DE EMERGÊNCIA - ABRAMEDE. Recomendações AMIB/ABRAMEDE de alocação de recursos em esgotamento durante a pandemia por Covid-19. Disponível em: https://crmsc.org.br/wp-content/uploads/2020/08/ Versa\%CC\%83o-2-0106-Protocolo-AMIB-de-alocac\%CC\%A7a\%CC\%83o-de-recursos-em-esgotamentodurante-a-pandemia-por-COVID.pdf. Acesso em: 4 abr. 2021.

48 Em sentido diverso, na Itália, afirmou-se: “Può rendersi necessario porre un limite di età all'ingresso in TI. Non si tratta di compiere scelte meramente di valore, ma di riservare risorse che potrebbero essere scarsissime a chi ha in primis più probabilità di sopravvivenza e secondariamente a chi può avere più anni di vita salvata, in un'ottica di massimizzazione dei benefici per il maggior numero di persone", em tradução livre: "Pode ser necessário estabelecer um limite de idade para entrar no TI. Não se trata apenas de fazer escolhas de valor, mas para reservar recursos que poderiam ser muito escassos para aqueles que têm maior probabilidade de sobrevivência e secundariamente para aqueles que podem ter mais anos de vida salvos, com vista à maximização benefícios para o maior número de pessoas" (VERGANO, Mario et al. Raccomandazioni di etica clinica per l'ammissione a trattamenti intensivi e per la loro sospensione. Roma: Società Italiana di Anestesia Analgesia Rianimazione e Terapia Intensiva, 2020. Disponivel em: https:// www.sicp.it/wp-content/uploads/2020/03/SIAARTI-Covid19-Raccomandazioni-di-etica-clinica.pdf. Acesso em: 4 abr. 2021).

49 "É com base nos direitos e princípios que regem o ordenamento brasileiro que se estabelece o dever de cuidar da pessoa idosa e garantir sua autonomia. Portanto, em tempos de escassez de recursos e falta de infraestrutura, ainda que seja desafiador proteger a pessoa humana, em especial idosos, é crucial promover tutela eficaz. Além disso, é preciso exigir da sociedade e do Estado olhar mais humano e atento às vulnerabilidades que emergem nesse contexto" (OLIVEIRA, Ana Sarah Vilela de; MACHADO, Juliana Campos; DADALTO, Luciana. Cuidados paliativos e autonomia de idosos expostos à Covid-19. Revista Bioética, Brasília, v. 28, n. 4, p. 595-603, dez. 2020). Como destacam Heloisa Helena Barboza e Vitor Almeida: "Diante da intrínseca vulnerabilidade da pessoa idosa potencializada pelas contingências existenciais naturais do processo de senescência, o direito prioritário à saúde emerge como instrumento indispensável para a promoção da proteção integral e do melhor interesse à luz do princípio da dignidade da pessoa humana. Desse modo, há de se cumprir o disposto no art. 15 do Estatuto do Idoso, que assegura o direito à saúde de modo integral, incluindo, inclusive, atenção especial às doenças que afetam preferencialmente 
O critério de idade somente pode ser levado em consideração a partir de uma análise mais complexa, que leve em conta outros fatores de triagem como potencial de recuperação ou de propagação da doença, caso no momento da avaliação haja evidências científicas suficientes, segundo critérios de saúde baseada em evidências para sua utilização. "A alocação de recursos baseados unicamente em ordens de chegada ou atendimento são inadequadas". ${ }^{50}$

\section{Considerações finais}

Observa-se no Brasil acentuada escalada do Covid-19, com falta de recursos na saúde. Neste cenário, a adoção de critérios éticos é de grande importância. A ordem de chegada não se mostra um critério adequado de alocação de recursos, salvo, hipoteticamente, entre pacientes com mesmo prognóstico. A idade dissociada de uma análise clínica não se mostra um critério válido.

Parâmetros decisórios devem estar predefinidos, orientados à maximização dos recursos, e sobretudo da busca por salvar o maior número de vidas. A exclusão de certos pacientes do acesso à UTI, ainda que dolorosa, deve ser seguida quando não houver a indicação clínica, ou se prioridades estiverem presentes, e não houver suficiência de leitos.

Pacientes com suspeita ou quadro clínico confirmado de Covid-19 não são melhores nem piores do que os outros. É importante atentar para necessidade de manter o fluxo de internações de outros quadros clínicos que demandem as internações, sem prioridade por doenças. De outro lado, em vista da possibilidade de contaminação, é preciso organizar os leitos de maneira a minimizar as contaminações hospitalares.

os idosos. Indispensável assegurar a efetividade do direito prioritário à saúde da pessoa idosa, de envergadura constitucional, decorrente do princípio do melhor interesse e do dever de cuidado e respeito à sua autonomia existencial, que devem ser realçados e promovidos, inclusive, em tempos de pandemia do novo coronavírus, na qual as taxas de letalidade atingem mais severamente a população idosa, além de provocar solidão e aprofundar as suas vulnerabilidades" (BARBOZA, Heloisa Helena; ALMEIDA, Vitor. A proteção das pessoas idosas e a pandemia do Covid-19: os riscos de uma política de "limpa-velhos". Migalhas, 17 abr. 2020).

50 ASSOCIAÇÃO DE MEDICINA INTENSIVA BRASILEIRA - AMIB. Recomendações da AMIB (Associação de Medicina Intensiva Brasileira), ABRAMEDE (Associação Brasileira de Medicina de Emergência, SBGG (Sociedade Brasileira de Geriatria e Gerontologia) e ANCP (Academia Nacional de Cuidados Paliativos) de alocação de recursos em esgotamento durante a pandemia por Covid-19. Disponivel em: https://www. amib.org.br/fileadmin/user_upload/amib/2020/abril/24/VJS01_maio_-_Versa_o_2_-_Protocolo_AMIB_ de_alocac_a_o_de_recursos_em_esgotamento_durante_a_pandemia_por_COVID.pdf. Acesso em: 4 abr. 2021; VERGANO, Mario et al. Raccomandazioni di etica clinica per l'ammissione a trattamenti intensivi e per la loro sospensione. Roma: Società Italiana di Anestesia Analgesia Rianimazione e Terapia Intensiva, 2020. Disponível em: https://www.sicp.it/wp-content/uploads/2020/03/SIAARTI-Covid19-Raccomandazioni-dietica-clinica.pdf. Acesso em: 4 abr. 2021. 
A Resolução CFM no 2.156/2016 estabelece critérios de admissão e alta em unidade de terapia intensiva, com critérios de priorização de pacientes. Quando tais critérios forem insuficientes, modelos de análise diagnóstica, a partir da ideia de justiça, beneficência, autonomia, melhor alocação dos recursos e maior chance de sobrevida, devem ser adotados.

$\mathrm{Na}$ priorização do quadro clínico, deve-se levar escalas preestabelecidas como preferenciais. Assim, pode-se colocar em conta aspectos como avaliação de expectativa, comorbidades, histórico clínico, para apontar a maior probabilidade de sobrevivência. Modelos como o Sofa podem oferecer indicativos importantes na adoção de padrões predefinidos de triagem.

Os critérios devem ser esclarecidos para as equipes, com a separação da triagem do atendimento.

Este é o parecer, salvo melhor juízo.

Informação bibliográfica deste texto, conforme a NBR 6023:2018 da Associação Brasileira de Normas Técnicas (ABNT):

SCHULMAN, Gabriel. Critérios para alocação de recursos escassos - leitos hos pitalares - em vista do Covid-19. Revista Brasileira de Direito Civil - RBDCivil, Belo Horizonte, v. 28, p. 231-253, abr./jun. 2021. Parecer. 